\title{
An EST-enriched Comparative Map of Brassica oleracea and Arabidopsis thaliana
}

\author{
Tien-Hung Lan, ${ }^{1}$ Terrye A. DelMonte, ${ }^{1}$ Kim P. Reischmann, ${ }^{1}$ Joel Hyman, ${ }^{1}$ \\ Stanley P. Kowalski, ${ }^{1,2}$ Jim McFerson, ${ }^{3,4}$ Stephen Kresovich, ${ }^{3,5}$ \\ and Andrew H. Paterson ${ }^{1,6}$ \\ ${ }^{1}$ Department of Soil and Crop Sciences, Texas A\&M University, College Station, Texas 77843 USA; ${ }^{3}$ Plant Genetic Resources \\ Unit, U.S. Department of Agriculture-Agricultural Research Service (USDA-ARS), Geneva, New York 14456 USA
}

\begin{abstract}
A detailed comparative map of Brassica oleracea and Arabidopsis thaliana has been established based largely on mapping of Arabidopsis ESTs in two Arabidopsis and four Brassica populations. Based on conservative criteria for inferring synteny, "one to one correspondence" between Brassica and Arabidopsis chromosomes accounted for $57 \%$ of comparative loci. Based on 186 corresponding loci detected in B. oleracea and $A$. thaliana, at least 19 chromosome structural rearrangements differentiate $B$. oleracea and $A$. thaliana orthologs. Chromosomal duplication in the $B$. oleracea genome was strongly suggested by parallel arrangements of duplicated loci on different chromosomes, which accounted for $41 \%$ of loci mapped in Brassica. Based on 367 loci mapped, at least 22 chromosomal rearrangements differentiate $B$. oleracea homologs from one another. Triplication of some Brassica chromatin and duplication of some Arabidopsis chromatin were suggested by data that could not be accounted for by the one-to-one and duplication models, respectively. Twenty-seven probes detected three or more loci in Brassica, which represent $25.3 \%$ of the 367 loci mapped in Brassica. Thirty-one probes detected two or more loci in Arabidopsis, which represent $23.7 \%$ of the 262 loci mapped in Arabidopsis. Application of an EST-based, cross-species genomic framework to isolation of alleles conferring phenotypes unique to Brassica, as well as the challenges and opportunities in extrapolating genetic information from Arabidopsis to Brassica and to more distantly related crops, are discussed.
\end{abstract}

Arabidopsis thaliana, a weed-like member of the Cruciferae family (tribe Sisymbrieae), offers many advantages for basic and applied plant research. These features include small stature, short life cycle, small genome size $(2 n=10$, estimated physical genome size of 100-120 Mb), low frequency of repetitive sequences ( $10 \%$ of the nuclear genome; Leutwiler et al. 1984), and prolific seed production. These features, combined with research of the past several decades yielding many mutants, efficient transformation systems, detailed genetic and physical maps, the availability of several P1, YAC, and BAC libraries, and 36,569 public ESTs (http:// www.cbc.umn.edu/ResearchProjects/Arabidopsis), make $A$. thaliana an ideal model for further molecular and genetic study (Meyerowitz and Somerville 1994). A multinational genome research initiative aiming to completely sequence the Arabidopsis genome by year 2004 (The Multinational Science Steering Committee 1997) is ahead of schedule. Such an accomplishment will undoubtedly create new scientific challenges and

Present addresses: ${ }^{2}$ USDA-ARS, Beltsville, Maryland 20704 USA; ${ }^{4}$ Washington Fruit Tree Research Commission, Wenatchee, Washington 98801 USA; ${ }^{5}$ Department of Plant Breeding and Biometry, Cornell University, Ithaca, New York 14850 USA; ${ }^{6}$ Applied Genetic Technology Center, Department of Crop and Soil Sciences, Department of Botany, and Department of Genetics, University of Georgia, Athens, Georgia 30602 USA.

${ }^{6}$ Corresponding author.

E-MAIL paterson@uga.edu; FAX (706) 583-0160. opportunities. One of the core issues will be how to apply the information obtained from the Arabidopsis genome project to the improvement of the world's leading crops.

The genus Brassica (tribe Brassiceae), including many important crops, is in the same taxonomic family as Arabidopsis thaliana. Such a close relationship suggests that crop plants of the genus Brassica will be among the earliest beneficiaries of a complete sequence of Arabidopsis. Economically, Brassica can be loosely categorized into oilseed, vegetable, and condiment crops. Brassica campestris, Brassica juncea, Brassica napus, and Brassica carinata provide $\sim 12 \%$ of the worldwide edible vegetable oil supplies (Labana and Gupta 1993) and generate $>\$ 8$ billion market value in North America and Europe. Brassica oleracea and B. campestris, the so-called "Cole crops," comprise a large variety of vegetables in our daily diet. Many of these vegetables have extreme morphological characteristics of basic interest, such as the enlarged inflorescence of cauliflower (B. oleracea subsp. botrytis) and broccoli (B. oleracea subsp. italica); enlarged stem of kohlrabi (B. oleracea subsp. gongylodes) and marrowstem kale (B. oleracea subsp. medullosa); enlarged root of turnip (B. campestris subsp. rapifera); enlarged and twisted leaves of Pak-choi (B. campestris subsp. chinesis) and Chinese cabbage (B. campestris subsp. pekinesis); and enlarged single apical 
bud of cabbage (B. oleracea subsp. capitata) or many axillary buds of Brussels sprouts (B. oleracea subsp. gemmifera) (Kalloo and Bergh 1993). Notably, although Arabidopsis is considered a close relative to Brassica, none of these phenotypes occur in Arabidopsis to nearly the same degree. Finally, Brassica nigra is primarily used as a condiment (mustard seed).

Through cytological study, the species relationship of crop Brassicas was described by the "triangle of U" (U 1935). Three allotetraploids, B. juncea $(2 n=36$, AABB), B. napus ( $2 n=38$, AACC), and B. carinata $(2 n=34$, $\mathrm{BBCC})$, originated through interspecific hybridization between different pairs of the three diploid species, $B$. nigra $(2 n=16, \mathrm{BB}), B$. oleracea $(2 n=18, \mathrm{CC})$, and $B$. campestris $(2 n=20, \mathrm{AA})$. Based on cytological examination and hybrid analysis, the haploid chromosome number of monogenomic species in the Brassiceae were found to range from 7 to 12 (Mizushima 1980). However, because of the available resolution of cytological techniques, detailed genomic relationships among monogenomic species were not fully revealed. Understanding the genomic relationship among monogenomic Brassica species will not only shed light on the evolution of the Brassica genome but also facilitate gene transfer among Brassica species. The rise of comparative mapping, the alignment of chromosomes based on common DNA markers, has provided the means to study in depth the parallels in genome structure and function of closely related species (Tanksley et al. 1988; Ahn and Tanksley 1993), and distantly related species (Paterson et al. 1996).

The present study aimed to better characterize the comparative genome organization of Brassica and Arabidopsis. Previous study of the genus Brassica showed that the proportion of low-copy DNA sequences was similar among diploid Brassica species, but a large number of rearrangements result in distinct chromosomal number and organization (Slocum et al. 1990; Landry et al. 1991, 1992; Song et al. 1991; Kianian and Quiros 1992; Lagercrantz and Lydiate 1996). Corresponding chromosomes in diploid and amphidiploid Brassica have been reported (Teutonico and Osborn 1994; Cheung et al. 1997a,b). Comparative mapping between Arabidopsis and Brassica revealed even more extensive chromosomal rearrangements (Kowalski et al. 1994a; Lagercrantz et al. 1996; Osborn et al. 1997). These studies, however, did not provide a complete scope of the genome comparison between Brassica and Arabidopsis because of the limited numbers of common markers. To address this issue, a larger number of markers were needed on the comparative maps. The present work, based on 186 corresponding loci detected, provides a much more detailed picture of the comparative genome organization of B. oleracea and A. thaliana. Furthermore, study of chromosomal duplication within the $B$. oleracea genome, based on 367 loci, illustrates some of the complexities that will be faced both in extrapolating Arabidopsis information to Brassica and in assembly of sequence-ready contigs for crop genomes.

\section{RESULTS}

\section{DNA Polymorphism}

Table 1 summarizes the DNA polymorphism detected by 200 Arabidopsis EST clones and 123 Brassica PstI genomic clones. The relatively low level of polymorphism in the $B$. oleracea $(\mathrm{RCB}) \times B$. oleracea ssp. alboglabra var.Bugh Kana $(\mathrm{BK}) \mathrm{F}_{2}$ population was consistent with the origin of RCB from B. oleracea ssp. alboglabra types (Song and Osborn 1992). The chance of detecting polymorphic probes is low and similar in both Arabidopsis crosses. There is variation in polymorphism rate associated with different restriction enzymes in Brassica, but no particular pattern is clear. In Arabidopsis, the restriction enzyme CfoI consistently detects more polymorphism than the other restriction enzymes, in both populations.

\section{Establishing Composite Linkage Maps}

\section{B. oleracea Linkage Maps}

Because many of the mapped polymorphisms were unique to one $B$. oleracea population, we constructed a composite linkage map for $B$. oleracea to more completely reflect all of the available comparative information. The assembly of the $B$. oleracea chromosome 1 composite map was illustrated in Figure 1 as an example, built according to the following rules: (1) Common loci detected in different populations could be identified based on the size of the restriction fragment from RCB, the common parent. These permitted the initial alignment of chromosomes of different populations. (2) The $\mathrm{RCB} \times \mathrm{GC}$ map was used as the primary linkage map because the largest number of loci were mapped in this population. Markers that did not detect

Table 1. Summary of the Polymorphism Detected by Arabidopsis EST Clones and Brassica Pstl Genomic Clones

\begin{tabular}{lccccc}
\hline \multicolumn{7}{c}{ Cfol } & Dral & EcoRI & EcoRV & HindIII \\
\hline Arabidopsis EST clones & & & & \\
RCB $\times$ GC & - & $67.3 \%$ & $73.2 \%$ & $75.0 \%$ & $54.0 \%$ \\
WS $\times$ M13 & $24.5 \%$ & - & $14.5 \%$ & $18.0 \%$ & $13.0 \%$ \\
WS $\times$ HM & $21.5 \%$ & - & $21.0 \%$ & $21.0 \%$ & $13.0 \%$ \\
Brassica Pstl clones & & & & \\
RCB $\times$ GC & - & $62.7 \%$ & $83.8 \%$ & $71.6 \%$ & $70.0 \%$ \\
RCB $\times$ PK & - & $54.2 \%$ & $73.0 \%$ & $69.7 \%$ & $44.7 \%$ \\
RCB $\times$ CAN & - & $85.3 \%$ & $69.1 \%$ & $89.9 \%$ & $93.5 \%$ \\
RCB $\times$ BK & - & $36.2 \%$ & $35.5 \%$ & $36.8 \%$ & $30.0 \%$ \\
\hline
\end{tabular}

Less than 80 EST clones were surveyed for RCB $\times$ BK, RCB $\times$ $C A N$, and $R C B \times P K$ and, thus were not included for comparison. 

BK
CAN
PK
$\operatorname{sGC}$
Composile

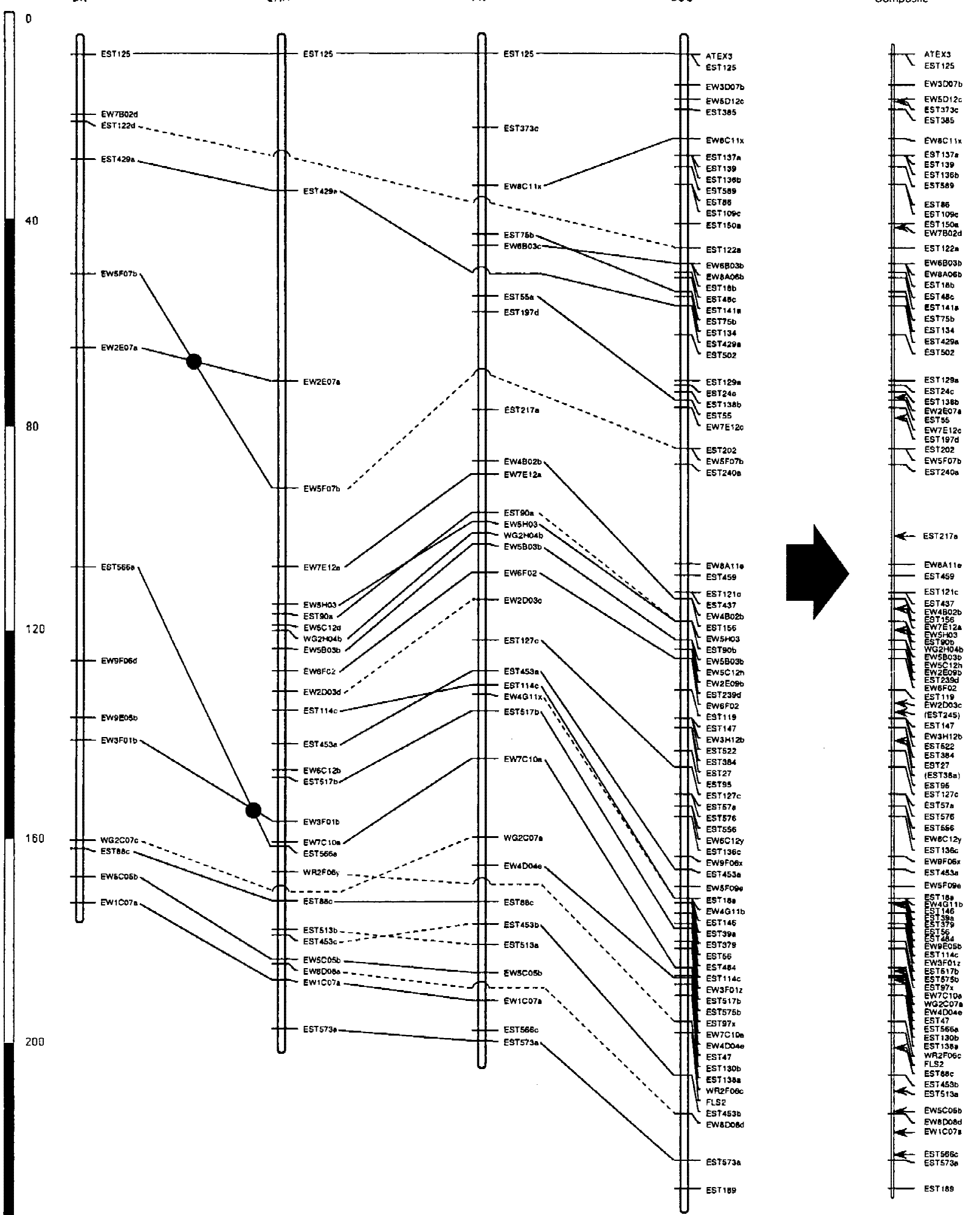

Figure 1 The assembly of the Brassica chromosome 1 composite map. Common loci (based on common restriction fragment sizes) were connected by solid lines, putatively homologous loci (with different restriction fragment sizes, but at corresponding sites) are connected by dashed lines. Filled circles placed on crossed lines indicate that respective orders of loci are statistically significantly different ( $\geq$ LOD 2.0) in the respective maps, suggesting possible chromosomal rearrangements. Arrows indicate the inferred locations of unique loci in the consensus map. 
Table 2. Possible Chromosomal Rearrangements Detected in Different Brassica Varieties

\begin{tabular}{lll}
\hline Chromosome & Marker interval & $\begin{array}{c}\text { Brassica } \\
\text { variety }\end{array}$ \\
\hline 1 & EW5F07b-EW2E07a & BK \\
2 & EST566a-EW3F01b & BK \\
7 & EW9F06a-EW8D10a & CAN \\
8 & EW7E01a-EW3A04b & PK \\
9 & EST517d-EST22a & CAN \\
9 & EW1D09b-EW5C12b & CAN \\
& EW5F07c-EW5H02 & CAN \\
& EST195a-EW5F07c & CAN \\
\hline
\end{tabular}

polymorphism in $\mathrm{RCB} \times \mathrm{GC}$ population but did detect polymorphism in other populations were mapped in other populations accordingly. For chromosome 8 , where the $\mathrm{RCB} \times \mathrm{GC}$ map exhibited few polymorphic markers, the $\mathrm{RCB} \times \mathrm{PK}$ linkage map was substituted. (3) The integration of unique loci was based on the closest common flanking loci, and the unique loci were positioned proportionally to their proximity to the flanking loci. (4) To test possible chromosomal rearrangements in different varieties, lod scores were calculated for the alternative (consensus) orders. Only if each possible consensus order in both populations could be ruled out by lod 2.0 was a rearrangement suggested.

The linkage maps span recombinational lengths of 743.0, 893.2, 947.1, and $871.3 \mathrm{cM}$ across the B. oleracea genome in $\mathrm{RCB} \times \mathrm{GC}, \mathrm{PK}, \mathrm{CAN}$, and $\mathrm{BK}$ populations, respectively, with an average length of $863.6 \mathrm{cM}$. The average recombinational lengths of $B$. oleracea chromosomes 1-9 are 189.2, 102.4, 91.7, 95.7, 97.4, 83.4, 77.2, 71.9 , and $72.9 \mathrm{cM}$, respectively. A total of 367 loci were detected in the composite map with an average interval between loci of $2.35 \mathrm{cM}$. Based on an estimated DNA content of $660 \mathrm{Mb}$ (Arumuganathan and Earle 1991), this corresponds to an average spacing of $1.8 \mathrm{Mb}$ between markers and suggests that most genes are within $0.9 \mathrm{Mb}$ of the nearest marker. Table 2 summarizes possible chromosomal rearrangements found among different $B$. oleracea varieties.

\section{A. thaliana Linkage Maps}

Construction of the A. thaliana composite map (Fig. 3, below) has been reported previously (Kowalski et al. 1994a), although this report includes 152 more loci. Specifically, common DNA polymorphisms detected on both populations served as "anchor loci" to infer the relative order of loci segregating in only one of the two populations. The map includes 262 loci across the A. thaliana genome. Thirty-one probes detect duplicated loci. Table 3 summarizes 20 duplicated loci newly detected by ESTs and cloned RAPD-amplified genomic DNA.

\section{Patterns of Correspondence of Brassica Chromosomes with One Another and with the Arabidopsis Chromosomes}

Figure 2 illustrates and Table 4 summarizes the composite linkage map of $B$. oleracea. We developed a model for the comparative organization of the chromosomes of $B$. oleracea and $A$. thaliana that assumes duplication of most Brassica chromosomes and one-toone correspondence of Brassica chromosomes with Arabidopsis chromosomes. The extent to which the observed data cannot be explained by this "null hypothesis," reflects the need for alternative hypotheses such as triplication of Brassica chromosomal segments or duplication of Arabidopsis chromosomal segments. The model was built based on the identification of SCEUS (smallest conserved evolutionary unit segments; O'Brien et al. 1993) of three or more loci that (1) maximize the number of corresponding DNA marker loci that are consistent with the model, (2) minimize the number of chromosomal rearrangements between duplicates (Brassica) or orthologs (Arabidopsis), (3) consider closely linked markers to be stronger evidence of synteny than distantly linked markers, and (4) consider a genetic distance of $>5 \mathrm{cM}$ to represent a true difference in locus order. This relatively large value was chosen to reflect not only the small size of the primary population but also the uncertainties associated with inference of loci mapped in other populations. Further constraints were imposed to evaluate the extent of du-

Table 3. Summary of Newly Detected Duplicated Loci in Arabidopsis Genome

\begin{tabular}{|c|c|c|c|c|c|}
\hline & $\mathrm{C} 1$ & $\mathrm{C} 2$ & $\mathrm{C} 3$ & $\mathrm{C} 4$ & $\mathrm{C} 5$ \\
\hline $\mathrm{C} 1$ & $\begin{array}{l}\text { EST168a,b } \\
\text { EST195a,b } \\
\text { EST78a,b }\end{array}$ & $\begin{array}{l}\text { EST46b,a } \\
\text { R379a,b }\end{array}$ & $\begin{array}{l}\text { EST36a,b } \\
\text { R5342f,a }\end{array}$ & EST21a,b & $\begin{array}{l}\text { EST150a,b } \\
\text { EST371b,a }\end{array}$ \\
\hline $\mathrm{C} 2$ & & & $\begin{array}{l}\text { EST30a,b } \\
\text { EST95b,a }\end{array}$ & $\begin{array}{l}\text { EST84c,a } \\
\text { EST } 8 b, a\end{array}$ & $\begin{array}{l}\text { EST151b,a } \\
\text { R6191cd,ce }\end{array}$ \\
\hline $\mathrm{C} 3$ & & & & & $\begin{array}{l}\text { EST138a,b } \\
\text { R4392b,a }\end{array}$ \\
\hline $\mathrm{C} 5$ & & & & & $\begin{array}{l}\text { EST240a,b } \\
\text { EST68a,b }\end{array}$ \\
\hline
\end{tabular}


C1

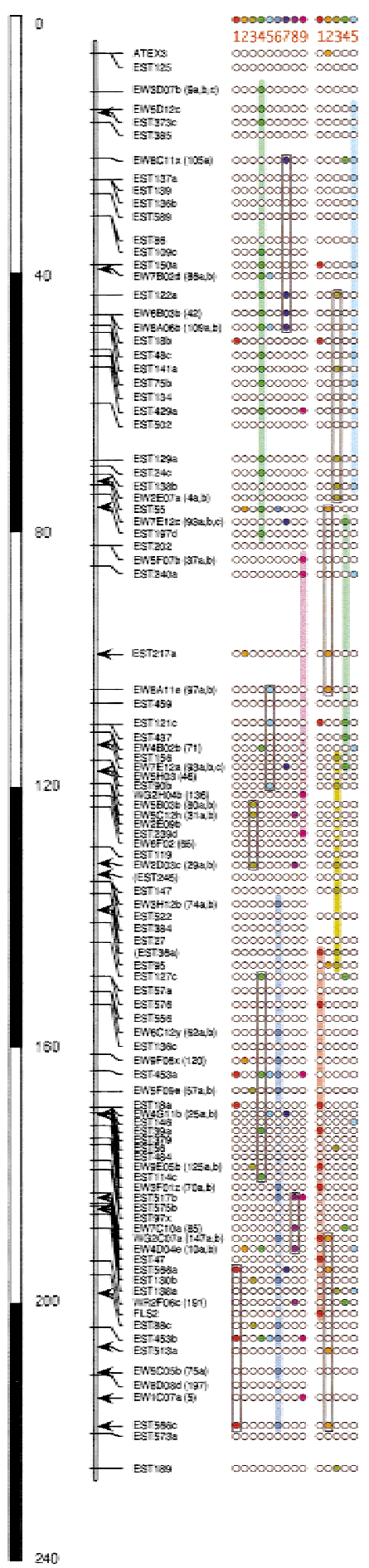

02

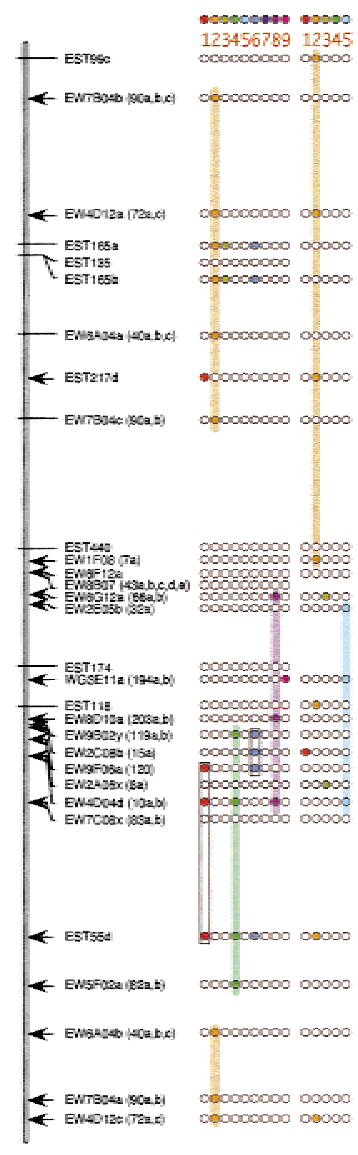

C3

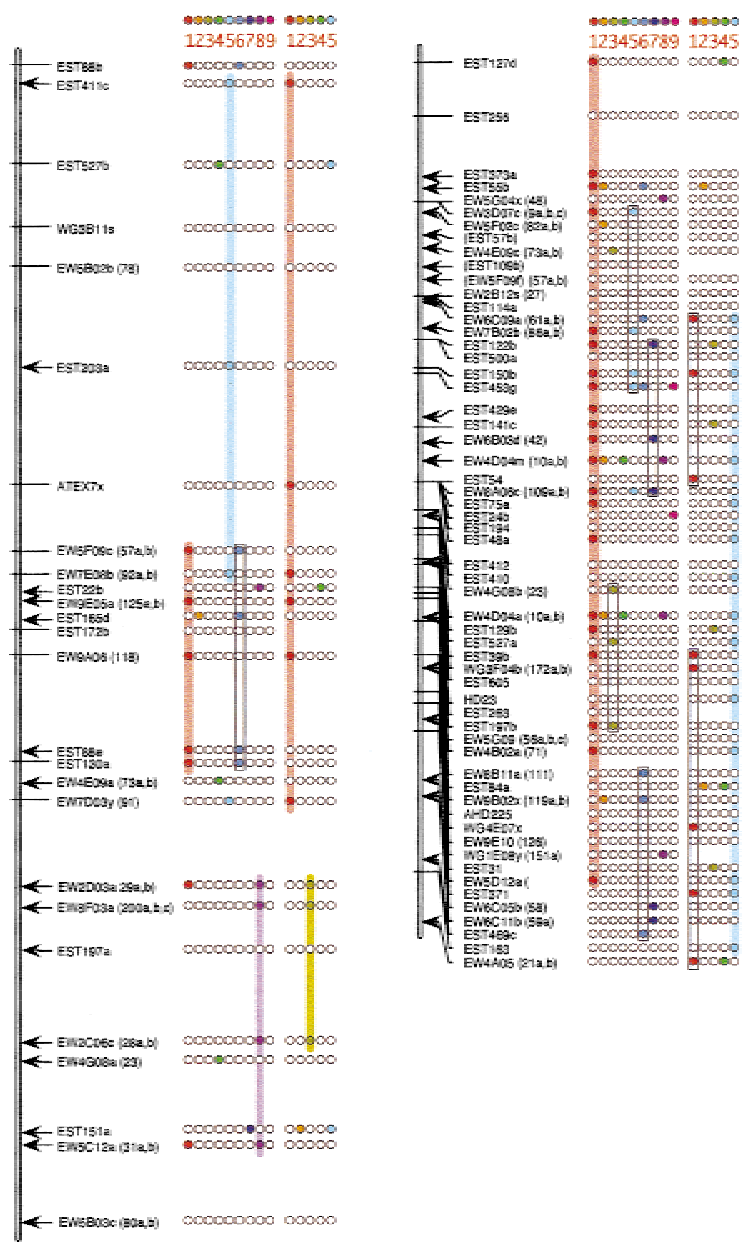

Figure 2 Composite RFLP linkage map of Brassica olearacea RCB $\times$ GC, RCB $\times$ CAN, RCB $\times$ PK and RCB $\times$ BK F2 Populations. Filled circles next to the loci indicate homoeologous Brassica loci (chromosomes 1-9, near right) or homologous Arabidopsis loci (chromosomes 1-5, far right) detected by the same probe. Open circles indicate that no polymorphism was detected for homoeologous (Brassica) and homologous (Arabidopsis) loci. A letter " $\mathrm{R}$ " next to the probe name indicates that the probe hybridizes to a repetitive DNA sequence in Arabidopsis. Specific colors are assigned to each homoeologous and homologous chromosome. Markers included in the duplication (Brassica) or one-to-one (Arabidopsis) models are connected by colored columns. Open columns indicate possible triplicated (Brassica) or duplicated (Arabidopsis) regions. 
C5

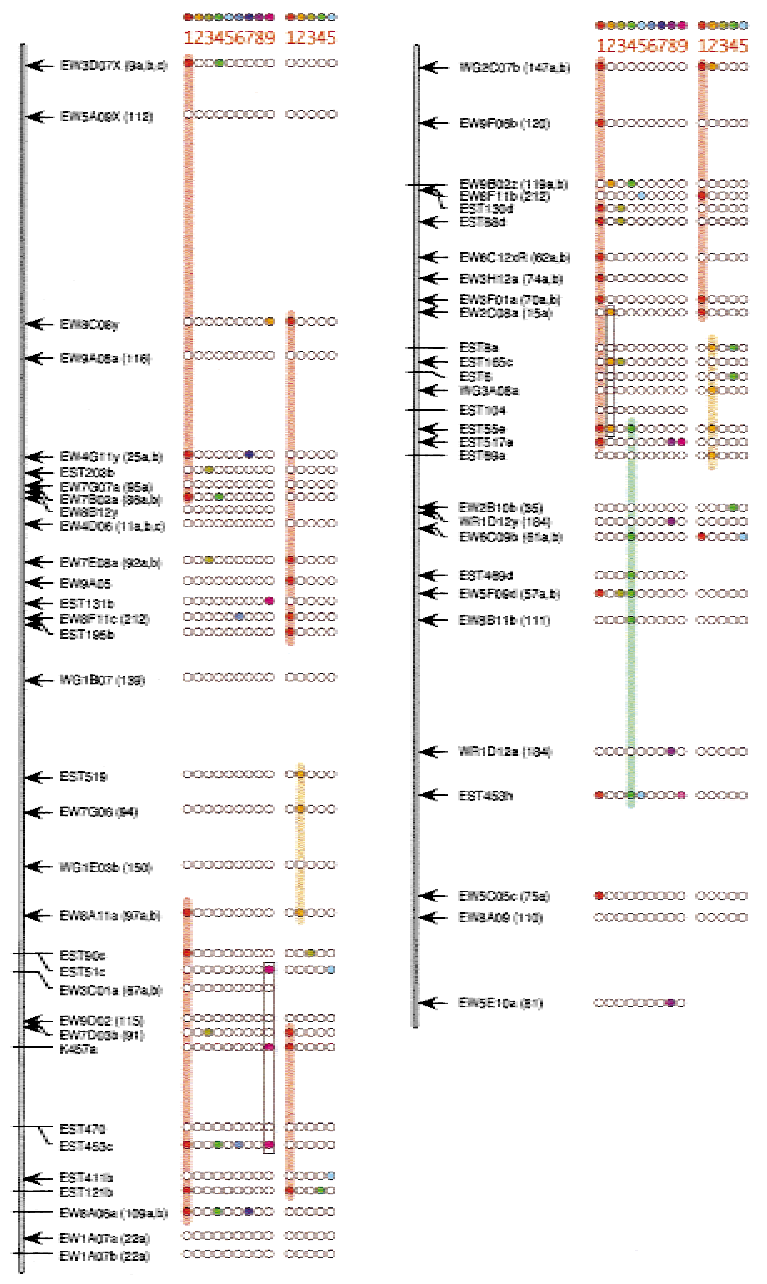

C7

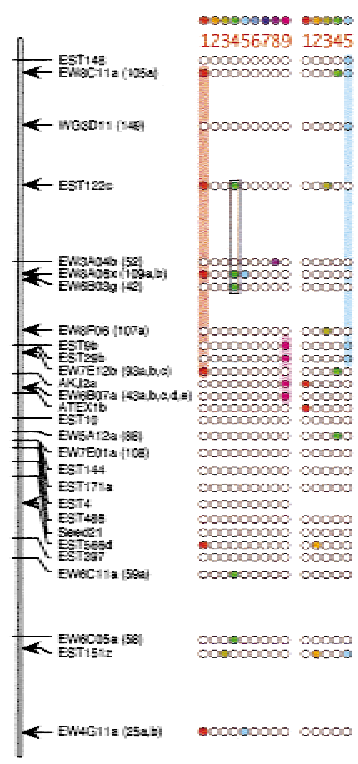

c8

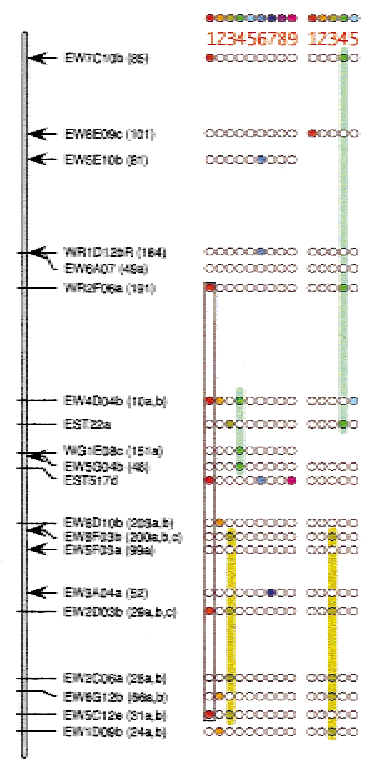

C9

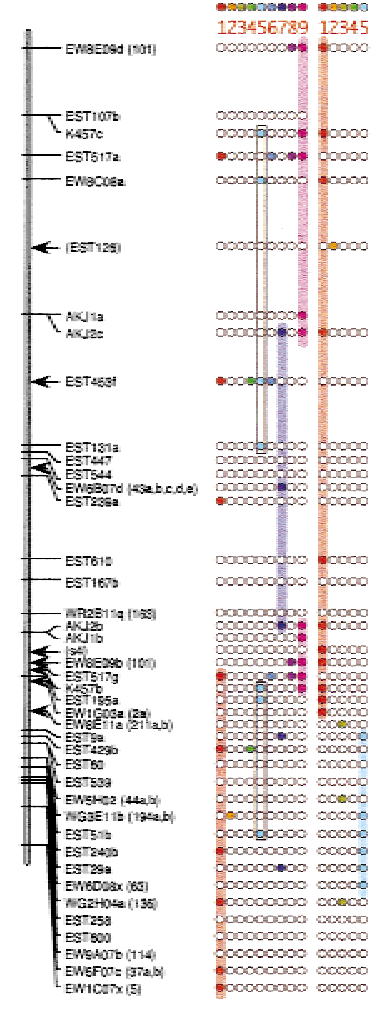

Figure 2 (Continued)

plication and triplication in Brassica. Specifically, possible regions of duplication along a chromosome were inferred first, in a manner that followed the above rules and did not allow different duplicated segments to overlap with each other by $>5 \mathrm{cM}$ (the threshold for inferring rearrangement). Finally, regions of possible triplication were inferred: These were allowed to overlap with duplicated segments but not with each other. From first principles, if the duplication process in Bras- sica were random (not associated with large chromosomal region), the duplication model would explain $12.5 \%$ of data (given that B. oleracea has nine chromosomes). The extent to which the model improves on this reflects the strength of evidence for duplication and triplication. By the same rationale, one-to-one correspondence of Brassica to Arabidopsis must account for significantly more than the random expectation of $25 \%$ of data to be meaningful. Higher levels of corre- 
Table 4. Summary of Brassica-Brassica and Brassica-Arabidopsis Correspondence

\begin{tabular}{|c|c|c|c|c|c|c|c|}
\hline \multirow[b]{2}{*}{ Chr. } & \multirow[b]{2}{*}{$\begin{array}{l}\text { No. of } \\
\text { loci }\end{array}$} & \multicolumn{3}{|c|}{ Brassica } & \multicolumn{3}{|c|}{ Arabidopsis } \\
\hline & & $\begin{array}{l}\text { no. of } \\
\text { corresponding } \\
\text { loci }\end{array}$ & $\begin{array}{c}\text { no. of } \\
\text { probes with } \\
\text { corresponding } \\
\text { loci } \\
\text { (avg/probe) }\end{array}$ & $\begin{array}{l}\text { no. }(\%) \text { of } \\
\text { corresponding } \\
\text { loci in model }\end{array}$ & $\begin{array}{c}\text { no. }(\%) \text { of } \\
\text { corresponding } \\
\text { loci }\end{array}$ & $\begin{array}{c}\text { no. of } \\
\text { probes with } \\
\text { corresponding } \\
\text { loci } \\
\text { (avg/probe) }\end{array}$ & $\begin{array}{l}\text { no. }(\%) \text { of } \\
\text { corresponding } \\
\text { loci in model }\end{array}$ \\
\hline 1 & 101 & 86 & $57(1.51)$ & $34(40 \%)$ & 55 & $47(1.17)$ & $26(47 \%)$ \\
\hline 2 & 30 & 29 & $19(1.53)$ & $16(55 \%)$ & 13 & $13(1.00)$ & $7(54 \%)$ \\
\hline 3 & 26 & 27 & $20(1.35)$ & $12(44 \%)$ & 13 & $12(1.08)$ & $9(69 \%)$ \\
\hline 4 & 58 & 54 & 34 (1.59) & $21(40 \%)$ & 30 & $25(1.20)$ & $13(43 \%)$ \\
\hline 5 & 33 & 24 & $16(1.50)$ & $8(32 \%)$ & 15 & $14(1.07)$ & $11(73 \%)$ \\
\hline 6 & 29 & 35 & $22(1.59)$ & 15 (44\%) & 14 & 11 (1.27) & $8(57 \%)$ \\
\hline 7 & 28 & 19 & $15(1.27)$ & $8(42 \%)$ & 15 & $13(1.15)$ & $5(33 \%)$ \\
\hline 8 & 20 & 23 & $17(1.35)$ & $7(30 \%)$ & 10 & $10(1.00)$ & $8(80 \%)$ \\
\hline 9 & 42 & 41 & $25(1.64)$ & 19 (46\%) & 21 & $21(1.00)$ & $17(81 \%)$ \\
\hline Total & 367 & 338 & $225(1.50)$ & $140(41 \%)$ & 186 & $166(1.12)$ & $106(57 \%)$ \\
\hline
\end{tabular}

spondence in small chromosomal regions may be suggestive of duplication of chromosomal segments.

\section{Brassica Chromosome 1}

The "duplication" model, in which Brassica chromosome 1 corresponds to nonoverlapping segments of Brassica chromosomes 4, 9, and 6 (sequentially, moving down the chromosome), explains only $40 \%$ of the additional loci detected by probes for which at least one locus mapped to chromosome 1. Loci that are not included in the duplication model occur in several closely linked clusters that suggest higher order redundancy of chromatin. In particular, 20 loci suggest correspondence to regions of chromosomes 7 (near top), 5 and 3 (nonoverlapping regions near middle), 4 (parallel to upper part of chromosome 6 correspondence), and 8 and 1 (nonoverlapping regions parallel to lower part of chromosome 6 correspondence), which represent possible "triplicated" chromosomal segments and account for $23 \%$ of the corresponding loci. Eight additional loci corresponding to chromosomes 3 and 9 (near the bottom) are noted but could not be inferred to be syntenic by the rules of our model.

One-to-one correspondence to regions of Arabidopsis chromosomes 5, 4, 3, and 1 (moving down the Brassica chromosomes) accounts for $47 \%$ of corresponding loci. Possible duplication in Arabidopsis is suggested by five loci corresponding to Arabidopsis chromosomes 2 (parallel to chromosome 5 correspondence) and 8 (parallel to chromosome 4 and 1 correspondence), accounting for $26 \%$ of the corresponding loci.

\section{Brassica Chromosome 2}

One-to-one correspondence suggests an internal duplication where the upper part of the chromosome (EW7B04b-EW7B04c) corresponds to the lower part of the chromosome (EW6A04b-EW4D12c), based on nine loci. The middle of chromosome 2 corresponds to chromosomes 8 and 4 . Overall, these data explain 55\% of the duplicated loci. Loci that are not included in this model suggest two possible segments corresponding to chromosomes 6 and 1 (parallel to chromosome 4 correspondence) and explain $21 \%$ of the corresponding loci.

One-to-one correspondence to regions of Arabidopsis chromosomes 2 and 5 accounts for 54\% of corresponding loci. Three additional loci on Arabidopsis chromosome 2 partially overlap the correspondence of Arabidopsis chromosome 5.

\section{Brassica Chromosome 3}

One-to-one correspondence to segments of Brassica chromosomes 5, 1, and 8 explains $38 \%$ of the duplicated loci. Loci that are not included in this model suggest a triplicated segment corresponding to chromosome 6 (parallel to chromosome 1 correspondence) and explain $14 \%$ of the corresponding loci. Three isolated loci corresponding to chromosome 4 are noted but cannot be accommodated by the rules of the model.

One-to-one correspondence to regions of Arabidopsis chromosomes 1 and 3 accounts for $69 \%$ of the corresponding loci.

\section{Brassica Chromosome 4}

One-to-one correspondence to a segment of Brassica chromosome 1 explains $43 \%$ of the duplicated loci. Loci that are not included in this model suggest triplicated regions corresponding to chromosome 5, 3, 6 , and 7 , which explain $17 \%$ of corresponding loci. Additional loci corresponding to chromosome 6 and 8 are noted but cannot be accommodated by the rules of the model.

One-to-one correspondence of Brassica chromosome 4 to Arabidopsis chromosome 5 explains $43 \%$ of 
the corresponding loci. Loci that are not included in this model suggest duplicated regions correspond to Arabidopsis chromosome 1 and explain 27\% of corresponding loci. Additional loci corresponding to chromosome 3 are noted but cannot be accommodated by the rules of the model.

\section{Brassica Chromosome 5}

One-to-one correspondence to Brassica chromosome 1 explains $32 \%$ of the duplicated loci. Three loci not included in this model suggest a triplicated region corresponding to chromosome 9 explaining $12 \%$ of the corresponding loci. Four loci corresponding to chromosome 4 are noted but cannot be accommodated by the rules of the model.

One-to-one correspondence to regions of Arabidopsis chromosomes 1 and 2 explains 73\% of the data.

\section{Brassica Chromosome 6}

One-to-one correspondence to segments of Brassica chromosomes 1 and 4 explains $44 \%$ of the duplicated loci. Loci that are not included in this model suggest a triplicated region corresponding to chromosome 2 and explain $9 \%$ of the corresponding loci. Isolated loci corresponding to chromosomes 2 and 8 are noted but cannot be accommodated by the rules of the model.

One-to-one correspondence to regions of Arabidopsis chromosomes 1 and 2 explains 57\% of the corresponding loci. Three loci corresponding to chromosome 4 are noted.

\section{Brassica Chromosome 7}

One-to-one correspondence to segments of Brassica chromosomes 1 and 9 explains $42 \%$ of the duplicated loci. Loci that are not included in the model suggest a triplicated region corresponding to chromosome 4 and explain $16 \%$ of corresponding loci.

One-to-one correspondence to a region of Arabidopsis chromosome 5 explains 33\% of the corresponding loci.

\section{Brassica Chromosome 8}

One-to-one correspondence to segments of Brassica chromosomes 4 and 3 explains 33\% of the duplicated loci. Loci that are not included in this model suggest a triplicated region corresponding to chromosome 1 and explain $21 \%$ of corresponding loci. Four loci corresponding to chromosome 2 and three loci corresponding to chromosome 6 are noted.

One-to-one correspondence to regions of Arabidopsis chromosomes 4 and 3 explains $80 \%$ of the corresponding loci.

\section{Brassica Chromosome 9}

One-to-one correspondence suggests an internal duplication of chromosome 9 where the chromosomal segment EW8E09d-AKJ2c corresponds to the segment
AKJ2b-K457b, involving 10 loci. An intervening region corresponds to chromosome 7, and the lower part of chromosome 9 corresponds to chromosome 1. Overall, these regions explain $46 \%$ of the duplicated loci. Loci that are not included in this model suggest triplicated regions corresponding to chromosome 5 and explain $16 \%$ of corresponding loci. Four loci corresponding to chromosome 8 and three loci corresponding to chromosome 6 are noted but cannot be accommodated by the rules of the model.

One-to-one correspondence to regions of Arabidopsis chromosomes 1 and 5 accounts for $81 \%$ of the corresponding loci. Three loci correspond to Arabidopsis chromosome 3 are parallel to chromosome 5 correspondence and may reflect duplication in Arabidopsis.

\section{Patterns of Correspondence of Arabidopsis Chromosomes with the Brassica Chromosomes}

The "one-to-one correspondence" model and duplication model were also tested on the Arabidopsis linkage map as well, which was illustrated in Figure 3 and summarized in Table 5.

\section{Arabidopsis Chromosome 1}

The one-to-one model, in which Arabidopsis chromosome 1 corresponds to nonoverlapping segments of Brassica chromosomes 5, 1, 4, and 9, explains 46\% of the loci detected by probes for which at least one locus mapped to chromosome 1 . Loci that are not included in the one-to-one model suggest a duplicated region corresponding to Brassica chromosome 3 (parallel to chromosome 5 correspondence), explaining 10\% of corresponding loci. Three loci corresponding to Brassica chromosome 6 are noted.

\section{Arabidopsis Chromosome 2}

The one-to-one model, in which Arabidopsis chromosome 2 corresponds to segments of Brassica chromosomes $1,5,1$, and 2, explains $52 \%$ of the loci. Loci that are not included in the model suggest a duplicated region corresponding to chromosome 6, explaining 15\% of corresponding loci.

\section{Arabidopsis Chromosome 3}

Our model suggests the correspondence of Arabidopsis chromosome 3 to nonoverlapping segments of Brassica chromosomes 1, 8, and 1 sequentially, explaining 49\% of the duplicated loci. Loci that are not included in the model suggest duplicated segments of chromosome 4 (near top) and 9 (near bottom), explaining 17\% of corresponding loci.

\section{Arabidopsis Chromosome 4}

The model suggests the correspondence of Arabidopsis chromosome 4 to Brassica chromosomes 8 and 4 and explains $36 \%$ of the loci. Loci that are not included in the model suggest a duplicated region corresponding 


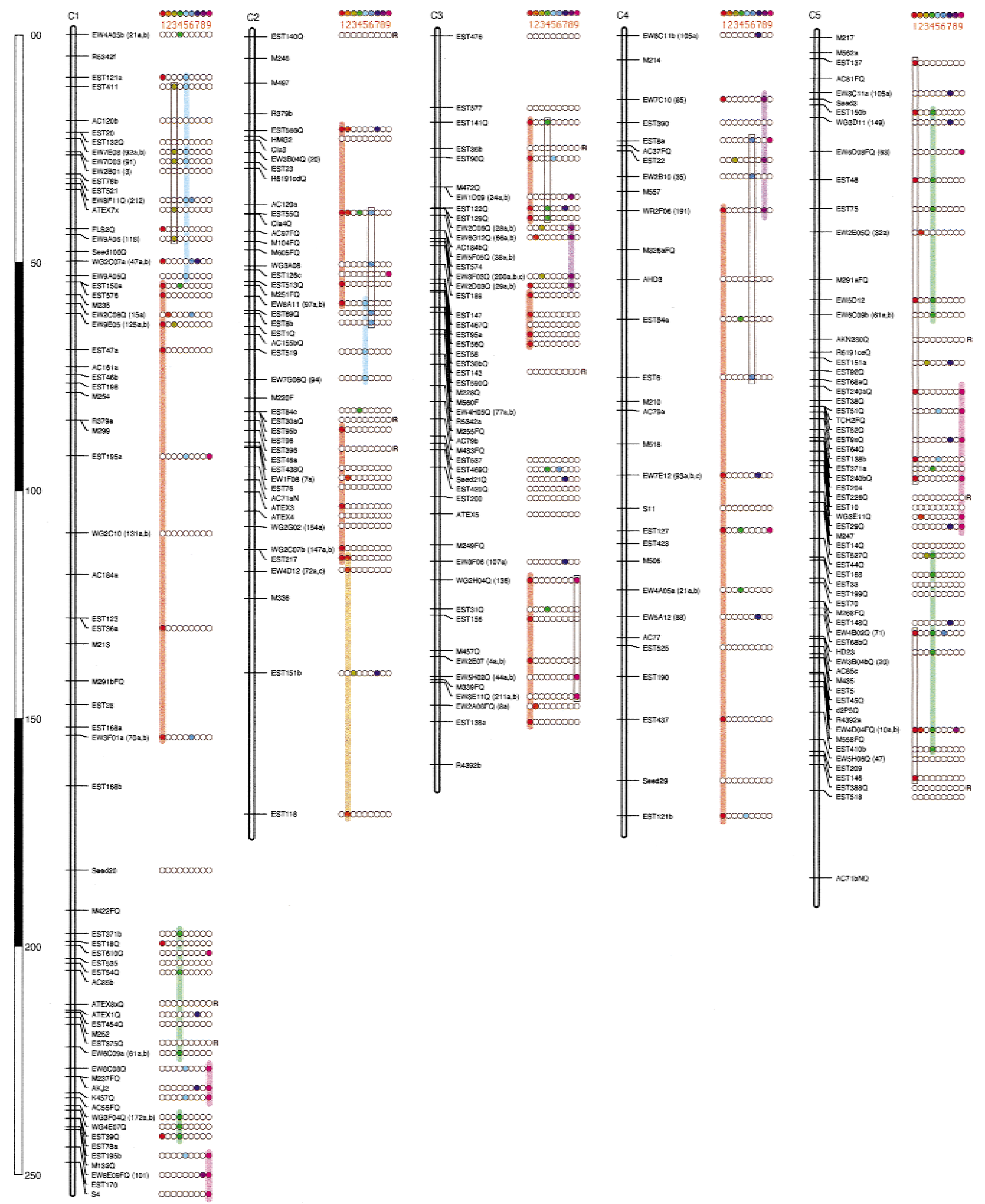

Figure 3 Composite RFLP linkage map of Arabidopsis thaliana HM x WS and M13 x WS F2 populations. Markers designated "FQ" are anchor loci, common to both populations. Markers mapped in the $\mathrm{HM} \times$ WS population are designated " $\mathrm{Q}$ ". The remaining markers were mapped in M13 x WS only. The construction of the $A$. thaliana composite map was as reported previously (Kowalski et al. 1994a). It should be noted that integration of data from two populations tends to inflate recombinational distance due to unequal recombination between populations. The filled circles next to the loci indicate homoeologous loci detected by the same probe on the Brassica composite map. Open circles indicate that no polymorphism was detected for homoeologous loci in RCB x GC Brassica populations. The letter "R" next to a probe name indicates that it hybridizes to a repetitive DNA sequence in the Brassica genome. Specific colors are asigned to each homoeologous chromosome. Markers included in the one-to-one model for Arabidopsis-Brassica correspondence are connected by filled columns. Open columns indicate possible duplicated regions in Brassica. 
Table 5. Summary of Arabidopsis-Brassica Correspondence

\begin{tabular}{lcccc}
\hline & & \multicolumn{3}{c}{ Arabidopsis } \\
\cline { 3 - 5 } Chr. & $\begin{array}{c}\text { No. of } \\
\text { loci }\end{array}$ & $\begin{array}{c}\text { no. of } \\
\text { corresponding } \\
\text { loci }\end{array}$ & $\begin{array}{c}\text { no. of probes with } \\
\text { corresponding loci } \\
\text { (avg/probe) }\end{array}$ & $\begin{array}{c}\text { no. (\%) of } \\
\text { corresponding } \\
\text { loci in model }\end{array}$ \\
\hline 1 & 70 & 52 & $34(1.53)$ & $24(46 \%)$ \\
2 & 47 & 27 & $19(1.42)$ & $14(52 \%)$ \\
3 & 51 & 35 & $24(1.46)$ & $17(49 \%)$ \\
4 & 30 & 22 & $14(1.57)$ & $8(36 \%)$ \\
5 & 64 & 44 & $27(1.63)$ & $17(39 \%)$ \\
& 262 & 180 & $118(1.53)$ & $80(44 \%)$ \\
\hline
\end{tabular}

to chromosome 6 , explaining $14 \%$ of corresponding loci. Three loci corresponding to chromosome 7 are noted.

\section{Arabidopsis Chromosome 5}

Our model suggests the correspondence of Arabidopsis chromosome 5 to segments of Brassica chromosomes 4,9 , and 4 and explains 39\% of the corresponding loci. Loci that are not included in the model suggest duplicated regions corresponding to chromosome 1, explaining $23 \%$ of the corresponding loci. Six loci corresponding to chromosome 7 are noted.

\section{DISCUSSION}

It is timely to consider the challenges and opportunities in extrapolating structural genomic information from Arabidopsis, the first plant for which the genome will be completely sequenced, to Brassica and other more distantly related plants.

Our model (Fig. 2) suggests that at least 22 chromosomal rearrangements differentiate the $B$. oleracea homologs from one another and at least 19 rearrangements differentiate $A$. thaliana from $B$. oleracea. In several instances the locations of chromosomal rearrangement breakpoints between Brassica homologs approximately match the locations of the breakpoints between Arabidopsis and Brassica. Some such instances include (1) Brassica chromosome 2, where the correspondence with Brassica chromosomes 2 and 8 breaks between EW7B04c and EW6G12a and the correspondence with Arabidopsis chromosomes 2 and 5 breaks between EW1F08 and EW2E05b; (2) Brassica chromosome 3, where the correspondence with Brassica chromosomes 1 and 8 breaks between EST130a and EW2D03a and the correspondence of Arabidopsis chromosomes 1 and 3 breaks between EW7D03y and EW2D03a; (3) Brassica chromosome 8, where the correspondence with Brassica chromosome 4 and 3 homologs breaks between EW5G04b and EST517d and the correspondence of Arabidopsis chromosomes 4 and 3 breaks between EST22a and EW8F03b; (4) Brassica chromosome 9, where the correspondence of Brassica chromosomes 9 and 1 breaks between K457b and EST517g and the correspondence of Arabidopsis chromosomes 1 and 5 breaks between EW1G03a and EST9a. Such rearrangement breakpoints that appear to be common to Brassica and Arabidopsis may reflect cases where both Arabidopsis and one Brassica homolog retain the chromosome organization of their common ancestor, whereas a duplicated Brassica homolog has undergone rearrangement. Similarly, chromosomal regions in which Arabidopsis gene order corresponds to one but not both Brassica homoeologs may reflect rearrangement of one Brassica homoeolog since duplication. For example, on Arabidopsis chromosome 5, the order for marker EW5D12, EST075, and EST150 is EST150-EST75EW5D12, and on Brassica chromosome 4, it follows the same order, but on Brassica chromosome 1, the order changes to EST75-EST150-EW5D12.

\section{Comparative Organization of Brassica Homoeologous Chromosomes}

The Brassica chromosomal duplication model explains $41 \%$ of the duplicated restriction fragment length polymorphism (RFLP) loci we mapped (Table 4). If there were no pattern to duplication, then the duplication would be expected to account for $<12.5 \%$ ( 1 out of 8 ) of data, because there are nine pairs of chromosomes in B. oleracea. Our data clearly indicate that duplication has involved large chromosome segments in Brassica. In a similar manner, if triplication accounts for more than an additional 14.3\% (1 out of 7 ) of data in Brassica, then it would be more common than expected to occur at random. Based on our model, triplication of Brassica chromosomal segments best explains $18 \%$ of the data, which is nominally greater than the expected value (14.3\%). Although the case for triplication is much weaker than for duplication, the clustering of triplicated loci into linked groups does tend to support prior suggestions based on smaller numbers of probes and isolated genomic regions (Kowalski et al. 1994a; Lagercrantz et al. 1996; Osborn et al. $1997)$ that some regions of the genome of $B$. oleracea (as well as B. rapa and B. nigra) may be triplicated. A 


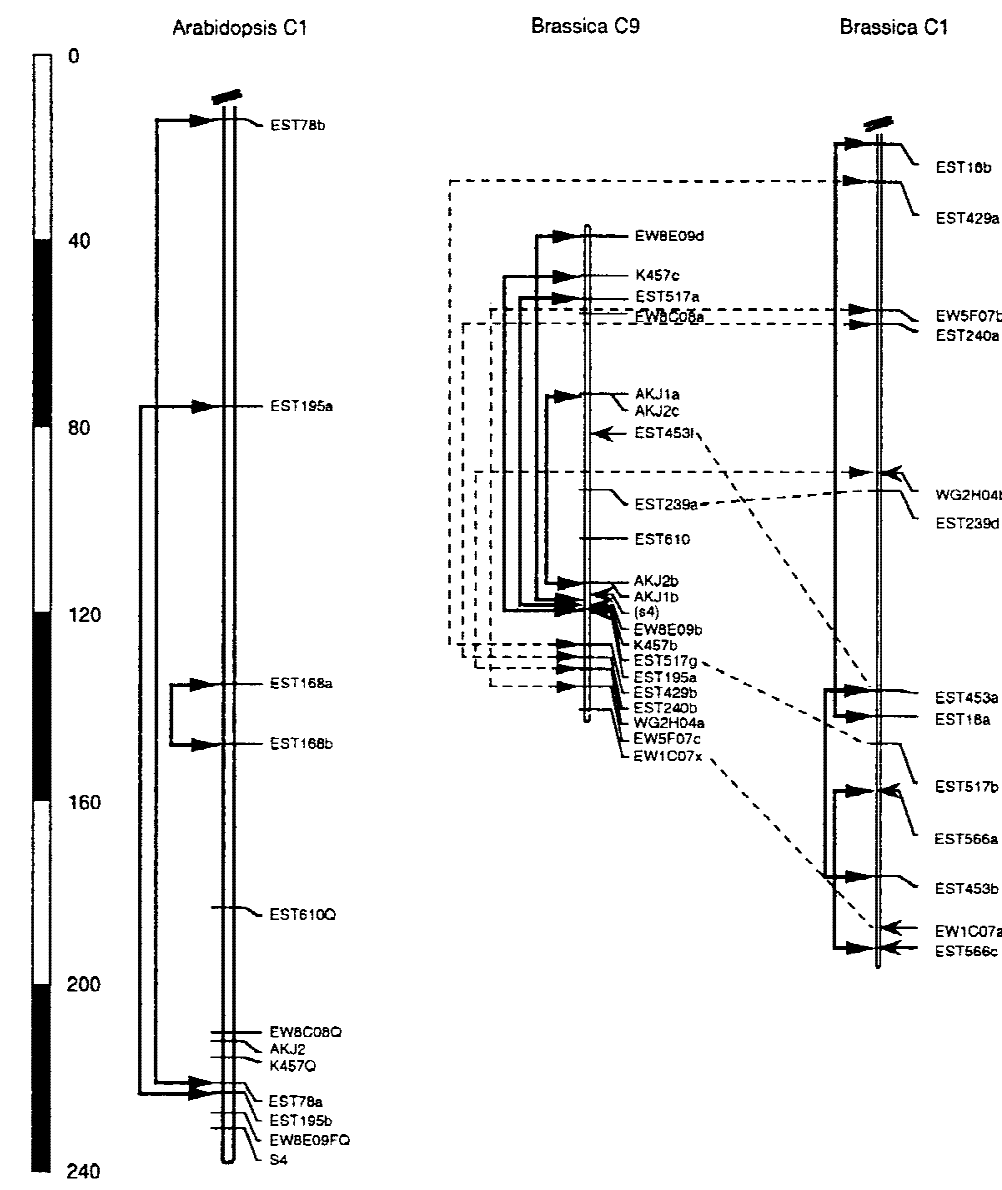

Figure 4 Intrachromosomal duplication of Arabidopsis chromosome 1, and an possible more-than-triplicated region of Brassica chromosome 1 and 9. Solid lines connect homoeologous loci (based on different restriction fragment sizes) located on the same chromosome. Dashed lines connect homoeologous loci located on different chromosomes.

fundamental problem in the use of genetic mapping data to evaluate duplication (and triplication) of chromatin is the need to detect DNA polymorphism. The assembly of physical maps for the Brassica genomes will alleviate this limitation but will require new methodology to efficiently determine the locus-specificity of BACs (or other large DNA clones) that hybridize to duplicated (or triplicated) probes.

\section{Alignment of Brassica and Arabidopsis Chromosomes}

The Brassica/Arabidopsis one-to-one correspondence model explain $57 \%$ of our observed data (Table 4). If the genomes of Brassica and Arabidopsis were randomly arranged with respect to one another, then one-to-one correspondence would account for $<20 \%$ ( 1 out of 5) of data in Arabidopsis. Our data clearly indicate extensive synteny of Brassica and Arabidopsis.

A total of 31 pairs of duplicated loci, including 20 pairs reported here for the first time (Table 3), mapped to A. thaliana, accounting for $23.7 \%$ of the loci de- tected. These duplicated loci expand on the earlier suggestion (Kowalski et al. 1994a) that part of the $A$. thaliana genome may have undergone ancient duplication. These ancient duplications could complicate contig-map construction and also could reduce the subset of Arabidopsis genes that are susceptible to "knockout" experiments (Sundaresan et al. 1995; Kempin et al. 1997). Notably, an intrachromosomal duplication appears to occur in A. thaliana chromosome 1 (Fig. 4).

Intrachromosomal duplication was observed in chromosomes 1,2 , and 9 of $\mathrm{B}$. oleracea (Fig. 5). Two independent studies on the genome of $B$. nigra reveal similar patterns on chromosome 5 (Truco and Quiros 1994) and chromosome 6 (Lagercrantz and Lydiate 1996), suggesting that such intrachromosomal duplication might be common in Brassica. If such intrachromosomal duplications preceded the duplication/ triplication of the ancestral $B$. oleracea genome, then even higher levels of duplication might be expected in modern B. oleracea. In our study, five probes did detect more than three segregating loci in B. oleracea, including EW4D04 (chromosomes 1, 2, 4, and 8), EW8A06 (chromosomes 1, 4, 5, and 7), EST55 (chromosomes 1, 2, 4, and 6), EST453 (chromosomes 1, 4, 5, 6, and 9), and EST517 (chromosomes 1, 6, 8, and 9). Although we cannot rule out the possibility that some of these more-than-triplicated loci might be the consequence of other duplication mechanisms, segments of Brassica chromosomes 1 and 9 did suggest the existence of such highorder chromosome segmental duplication (Fig. 5). More probes mapped in this region should provide further evidence.

Through comparative mapping, many powerful tools already created for Arabidopsis can now be applied to Brassica. For example, Arabidopsis cDNA sequences may be used to isolate homologous genes in Brassica, Arabidopsis BAC/YAC contigs may be used in Brassica for map-based cloning, and Arabidopsis highresolution maps may help to resolve clustered markers in Brassica (Liu et al. 1996). Arabidopsis genomic tools may guide the isolation of Brassica alleles conferring unique phenotypes. Brassica and Arabidopsis may have diverged as little as 10 mya (Muller 1981), suggesting that $\sim 90 \%$ of chromosomal segments $<5 \mathrm{cM}$ may remain colinear (Paterson et al. 1996). A comparative map with a density of $<5 \mathrm{cM} /$ marker makes it relatively easy to evaluate correspondence of Brassica quantitative trait loci (QTLs) to Arabidopsis mutations or can- 


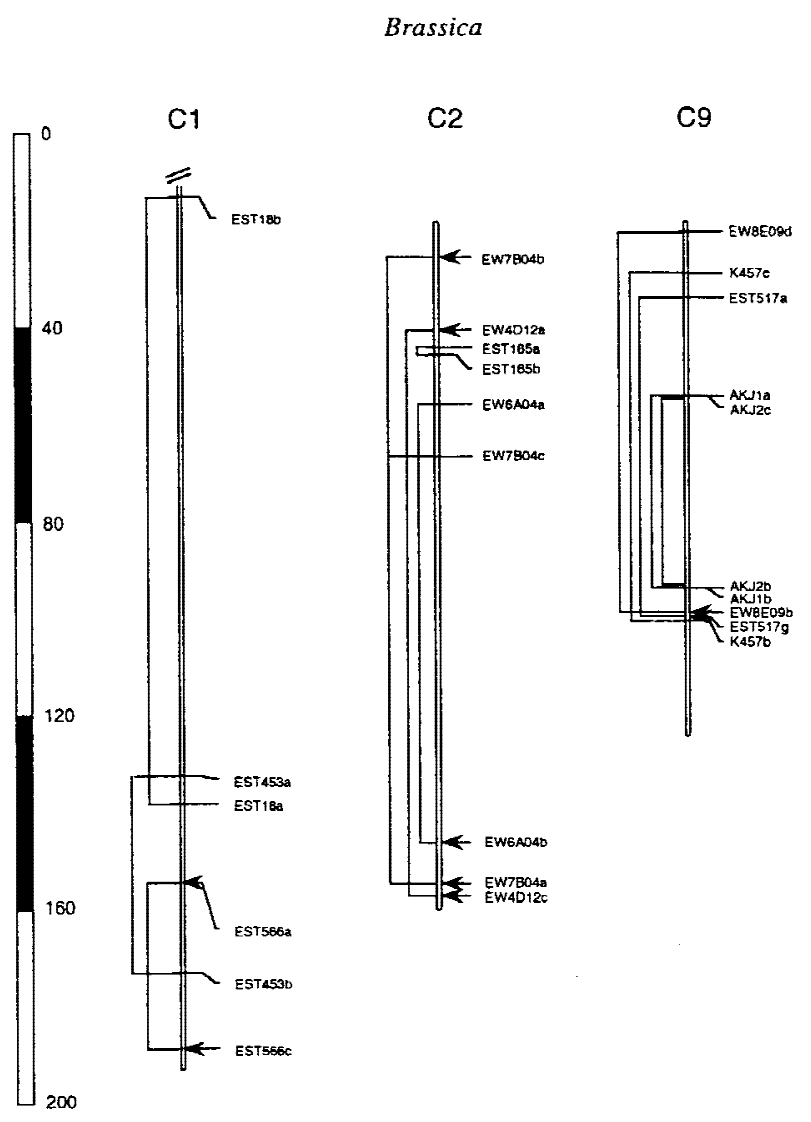

Figure 5 Intrachromosomal duplication detected by three or more duplicate loci in Brassica.

didate genes. Furthermore, a comparative map of $B$. oleracea (CC genome) and $A$. thaliana can be extended to an amphidiploid species of Brassica such as B. napus (AACC genome), where genome complexity is redoubled.

Genetic linkage maps based on ESTs (Berry et al. 1995) enable one to use sequence information to screen for conservation with distantly related taxa. For example, disease-resistance-like ESTs could be potentially useful in locating disease-resistance loci in a specifically designed segregating population other than Arabidopsis (Botella et al. 1997). Also, through selection of highly conserved ESTs, comparative organization of the chromosomes of even distantly related species such as Arabidopsis, Gossypium (cotton), and Sorghum can be studied using the same probes (Paterson et al. 1996). Thus, a cross-genome comparative map based on a common set of ESTs may eventually provide a direct comparison of macro- and microcolinearity across various species. The combination of ESTs and DNA microarray technology (Winzeler et al. 1998) could accelerate this process. Furthermore, mapping the common set of ESTs to Arabidopsis megabase DNA libraries (Schmidt et al. 1995; Zachgo et al. 1996; Agyare et al. 1997) will extend the Arabidopsis physical map and
DNA contigs to other plants. Thus, using Arabidopsis contigs to assist map-based cloning in cotton, sorghum, or other genomes may be more feasible. Such a cross-genome framework and toolbox could profoundly affect future genome sequencing projects in related taxa. It is of interest not only to elucidate the portions of genome that are conserved (common) among various species but also the portions that are divergent among species. Thus, the priority of subsequent crop genome sequencing projects might be focused on genomic regions that are poorly conserved, so that scarce financial resources are used more efficiently.

\section{METHODS}

\section{Plant Materials}

Two $A$. thaliana $\mathrm{F}_{2}$ populations were used in this study: $A$. thaliana ecotype Wassilewskija (WS) $\times$ mutant stock M13 (Liu et al. 1996) and WS $\times$ Hannover/Münden (HM) (Kowalski et al. 1994b). Subsets of 78 individuals from each population were used for mapping Arabidopsis ESTs. Four B. oleracea $\mathrm{F}_{2}$ mapping populations were used in this experiment: RCB (selfcompatible) $\times$ B. oleracea var. Green Comet (USDA collection, accession no. G30771, from North America), RCB $\times$ B. oleracea var. Cantanese (USDA collection, accession no. PI462224, originally from Italy), $\mathrm{RCB} \times B$. oleracea var.Pusa Katki (USDA collection, accession no. PI274783, originally from India), and RCB $\times B$. oleracea var.Bugh Kana (USDA collection, accession no. PI249556, originally from Thailand), composed of 56, 247, 250, and 246 individuals, respectively. A. thaliana seed were obtained from the Arabidopsis Biological Resources Center at Ohio State University, directed by Dr. R.L. Scholl. Rapid-cycling Brassica was from the Crucifer Genetics Cooperative, Madison, WI. Seed and pollen of other B. oleracea varieties were generously provided by Dr. J. McFerson and Dr. S. Kresovich, then at USDA-ARS, Geneva, NY.

\section{Genotyping}

DNA extraction, electrophoresis, Southern blotting and autoradiography were as described previously (Kowalski et al. 1994a). A total of 113 Brassica PstI genomic clones ("EW," "WG," and "WR," from Pioneer HiBred), 35 Arabidopsis genomic clones ("M," from Dr. E. Meyerowitz, Caltech), 23 Arabidopsis anonymous cDNA clones ("AC," "ATEX," and "TCH"), four cloned RAPD-PCR products ("R;" unpubl.), 198 Arabidopsis EST clones ("EST," from Dr. R.L. Scholl, the Arabidopsis Biological Resources Center, Ohio State University), and 19 putatively embryo-specific Arabidopsis EST clones ("AHD," “AKJ," “AKN," “Cla," "d2P," “FLS," "HD," "HMG," "K," "S," and "Seed," from Dr. Terry L. Thomas, Texas A\&M University) were used in this study.

\section{Data Analysis}

RFLP linkage maps were constructed using MapMaker (Lander et al. 1987). Linkage groups were built at threshold of lod (logarithm of odds)=2.1 for A. thaliana and lod=2.5 for B. oleracea. Genetic distances (in centiMorgans) were calculated using the Kosambi mapping function. 


\section{ACKNOWLEDGMENTS}

We thank Tzung-Fu Hsieh for critical discussion, Kenneth Feldmann and JoVan Currie for technical help, the Texas Higher Education Coordinating Board, USDA Plant Genome Program, and Texas Agricultural Experimental Station for funding. We thank Pioneer HiBred Production, Ltd. for providing a subset of the DNA probes used.

The publication costs of this article were defrayed in part by payment of page charges. This article must therefore be hereby marked "advertisement" in accordance with 18 USC section 1734 solely to indicate this fact.

\section{REFERENCES}

Agyare, F.D., D.A. Lashkari, A. Lagos, A.F. Namath, G. Lagos et al. 1997. Mapping expressed sequence tag sites on yeast artificial chromosome clones of Arabidopsis thaliana DNA. Genome Res. 7: 1-9.

Ahn, S. and S.D. Tanksley. 1993. Comparative linkage maps of the rice and maize genomes. Proc Natl. Acad. Sci. 90: 7980-7984.

Arumuganathan, K. and E.D. Earle. 1991. Estimation of nuclear DNA content of plants by flow cytometry. Plant Mol. Biol. Rep. 9: 208-218.

Berry, R., T.J. Stevens, N.A.R. Walter, A.S. Wilcox, T. Rubano et al. 1995. Gene-based sequence-tagged-sites (STSs) as the basis for a human gene map. Nat. Genet. 10: 415-423.

Botella, M.A., M.J. Coleman, D.E. Hughes, M.T. Nishimura, J.D.G. Jones et al. 1997. Map positions of 47 Arabidopsis sequences with sequence similarity to disease resistance genes. Plant J. 12: 1197-1211.

Cheung, W.Y., G. Champagne, N. Hubert, and B.S. Landry. 1997a. Comparison of the genetic maps of Brassica napus and Brassica oleracea. Theor. Appl. Genet. 94: 569-582.

Cheung, W.Y., L. Friesen, G.F.W. Rakow, G. Seguin Swartz, and B.S. Landry. 1997b. A RFLP-based linkage map of mustard (Brassica juncea (L.) Czern. and Coss.). Theor. Appl. Genet. 94: 841-851.

Kalloo, G. and B.O. Bergh. 1993. Genetic improvement of vegetable crops. Pergamon Press, Oxford, UK.

Kempin, S.A., S.J. Liljegren, L M. Block, S.D. Rounsley, M.F. Yanofsky et al. 1997. Targeted disruption in Arabidopsis. Nature 389: 802-803.

Kianian, S.F. and C.F. Quiros. 1992. Generation of a Brassica oleracea composite RFLP map: linkage arrangements among various populations and evolutionary implications. Theor. Appl. Genet. 84: $544-554$.

Kowalski, S.P., T.-H. Lan, K.A. Feldmann, and A.H. Paterson. 1994a. Comparative mapping of Arabidopsis thaliana and Brassica oleracea chromosomes reveals islands of conserved organization. Genetics 138: 499-510.

.1994b. QTL mapping of naturally-occurring variation in flowering time of Arabidopsis thaliana. Mol. \& Gen. Genet. 245: 548-555.

Labana, K.S. and M.L. Gupta. 1993. Importance and origin. In Breeding oilseed Brassicas (eds. K.S. Labana, S.S. Banga, and S.K. Banga), pp. 1-20. Spinger-Verlag Press, Berlin, Germany.

Lagercrantz, U. and D J. Lydiate. 1996. Comparative genome mapping in Brassica. Genetics 144: 1903-1910.

Lagercrantz, U., J. Putterill, G. Coupland, and D. Lydiate. 1996. Comparative mapping in Arabidopsis and Brassica, fine scale genome collinearity and congruence of genes controlling flowering time. Plant J. 9: 13-20.

Lander, E., P. Green, J. Abrahamson, A. Barlow, M. Daly et al. 1987. MAPMAKER: An interactive computer package for constructing primary genetic linkage maps of experimental and natural populations. Genomics 1: 174-181.

Landry, B.S., N. Hubert, and T. Etoh. 1991. A genetic map for Brassica napus based on restriction fragment length polymorphisms detected with expressed DNA sequences. Genome 34: $543-552$.
Landry, B.S., N. Hubert, and R. Crete. 1992. A genetic map of Brassica oleracea based on RFLP markers detected with expressed DNA sequences and mapping resistance genes to race 2 of Plasmodiophora brassicae (Woronin). Genome 35: 409-419.

Leutwiler, L., B. Hough-Evans, and E. Meyerowitz. 1984. The DNA of Arabidopsis thaliana. Mol. \& Gen. Genet. 219: 225-234.

Liu, S.C., S.P. Kowalski, T.H. Lan, K.A. Feldmann, and A.H. Paterson. 1996. Genome-wide high-resolution mapping by recurrent intermating using Arabidopsis thaliana as a model. Genetics 142: $247-258$.

Meyerowitz, E.M. and C.R. Somerville. 1994. Arabidopsis. Cold Spring Harbor Laboratory Press, Cold Spring Harbor, NY.

Mizushima, U. 1980. Genome analysis in Brassica and allied genera. In Brassica crops and wild allies (eds. S. Tsunoda, K. Hinata, and C. Gomez-Campo), pp. 89-106. Japan Scientific Societies Press, Tokyo, Japan.

Muller, J. 1981. Fossil pollen records of extent angiosperms. Bot. Rev. 47: 1-142.

The Multinational Science Steering Committee. 1997. The multinational coordinated Arabidopsis thaliana genome research project. Progress report: Year six. National Science Foundation.

O’Brien, S.J., J.E. Womack, L.A. Lyons, K.J. Moore, N.A. Jenkins et al. 1993. Anchored reference loci for comparative genome mapping in mammals. Nat. Genet. 3: 103-112.

Osborn, T.C., C. Kole, I.A.P. Parkin, A.G. Sharpe, M. Kuiper et al. 1997. Comparison of flowering time genes in Brassica rapa, $B$. napus and Arabidopsis thaliana. Genetics 146: 1123-1129.

Paterson, A.H., T.-H. Lan, K.P. Reischmann, C. Chang, Y.-R. Lin et al. 1996. Toward a unified map of higher plant chromosomes, transcending the monocot-dicot divergence. Nature Genet. 14: $380-382$.

Schmidt, R., J. West, K. Love, Z. Lenehan, C. Lister et al. 1995. Physical map and organization of Arabidopsis chromosome 4. Science 270: $480-483$.

Slocum, M.K., S.S. Figdore, and W.C. Kennard. 1990. Linkage arrangement of restriction fragment length polymorphism loci in Brassica oleracea. Theor. Appl. Genet. 80: 57-64.

Song, K. and T.C. Osborn. 1992. Polyphyletic origins of Brassica napus : New evidence based on organelle and nuclear RFLP analyses. Genome 35: 992-1001.

Song, K., J. Suzuki, M. Slocum, and T.C. Osborn. 1991. A linkage map of Brassica rapa (syn. campestris) based on restriction fragment length polymorphism loci. Theor. Appl. Genet. 82: 296-304.

Sundaresan, V., P. Springer, T. Volpe, S. Haward, J. Jones et al. 1995. Patterns of gene action in plant development revealed by enhancer trap and gene trap transposable elements. Genes \& Dev. 9: 1797-1810.

Tanksley, S.D., R. Bernatzky, and N.L. Lapitan. 1988. Conservation of gene repertoire but not gene order in pepper and tomato. Proc. Natl. Acad. Sci.85: 6419-6423.

Teutonico, R.A. and T.C. Osborn. 1994. Mapping of RFLP and qualitative trait loci in Brassica rapa and comparison to the linkage maps of B. napus, B. oleracea, and Arabidopsis thaliana. Theor. Appl. Genet. 89: 885-894.

Truco, M J. and C.F. Quiros. 1994. Structure and organization of the B genome based on a linkage map in Brassica nigra. Theor. Appl. Genet. 89: 590-598.

U, N. 1935. Genome analysis in Brassica with special reference to the experimental formation of $B$. napus and peculiar mode of fertilization. Jpn. J. Bot. 7: 389-452.

Winzeler, E.A., D.R. Richards, A.R. Conway, A.L. Goldstein, S. Kalman et al. 1998. Direct allelic variation scanning of the yeast genome. Science 21: 1194-1197.

Zachgo, E.A., M L. Wang, J. Dewdney, D. Bouchez, C. Comilleri et al. 1996. A physical map of chromosome 2 of Arabidopsis thaliana. Genome Res. 6: 19-25.

Received August 18, 1999; accepted in revised form March 27, 2000. 


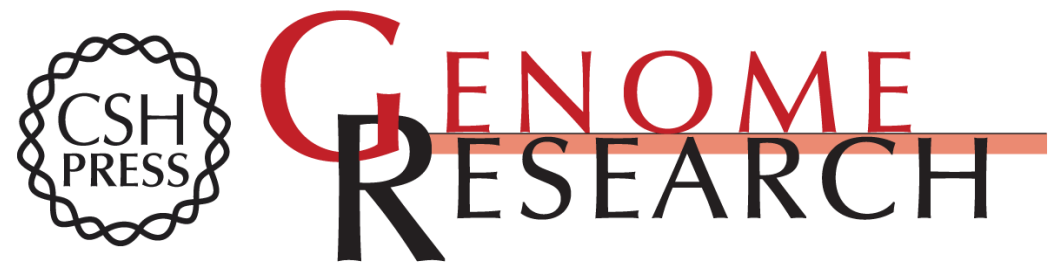

\section{An EST-enriched Comparative Map of Brassica oleracea and Arabidopsis thaliana}

Tien-Hung Lan, Terrye A. DelMonte, Kim P. Reischmann, et al.

Genome Res. 2000 10: 776-788

Access the most recent version at doi:10.1101/gr.10.6.776

References This article cites 31 articles, 10 of which can be accessed free at:

http://genome.cshlp.org/content/10/6/776.full.html\#ref-list-1

\section{License}

Email Alerting Receive free email alerts when new articles cite this article - sign up in the box at the Service top right corner of the article or click here.

\section{Affordable, Accurate Sequencing.}

